\title{
The space-time outside a source of gravitational radiation: the axially symmetric null fluid
}

\author{
L. Herrera ${ }^{1,2, a}$, A. Di Prisco ${ }^{1, b}$, J. Ospino ${ }^{3, c}$ \\ ${ }^{1}$ Escuela de Física, Facultad de Ciencias, Universidad Central de Venezuela, Caracas, Venezuela \\ 2 Instituto Universitario de Física Fundamental y Matemáticas, Universidad de Salamanca, Salamanca, Spain \\ ${ }^{3}$ Departamento de Matemática Aplicada and Instituto Universitario de Física Fundamental y Matemáticas, \\ Universidad de Salamanca, Salamanca, Spain
}

Received: 13 September 2016 / Accepted: 24 October 2016 / Published online: 4 November 2016

(C) The Author(s) 2016. This article is published with open access at Springerlink.com

\begin{abstract}
We carry out a study of the exterior of an axially and reflection symmetric source of gravitational radiation. The exterior of such a source is filled with a null fluid produced by the dissipative processes inherent to the emission of gravitational radiation, thereby representing a generalization of the Vaidya metric for axially and reflection symmetric space-times. The role of the vorticity, and its relationship with the presence of gravitational radiation is put in evidence. The spherically symmetric case (Vaidya) is, asymptotically, recovered within the context of the $1+3$ formalism.
\end{abstract}

\section{Introduction}

It is well known that in the hydrodynamic description of a physically meaningful source (i.e. bounded and regular) of gravitational radiation, there should be present a dissipative term, which accounts for the increasing of entropy associated to such an emission $[1,2]$. Accordingly, we should expect that any exterior of such a source should entail the presence of incoherent radiation (null fluid), associated to those irreversible processes.

It is our purpose in this manuscript to provide a full description of the space-time surrounding a bounded source of gravitational radiation. For simplicity we shall impose the highest degree of symmetry compatible with the presence of gravitational radiation, i.e. axially and reflection symmetry. Thus, the exterior of such a source is filled with a null fluid, and it represents a generalization of the Vaydia metric for axially and reflection symmetric space-times.

\footnotetext{
a e-mail: 1herrera@usal.es

b e-mail: alicia.diprisco@ciens.ucv.ve

c e-mail: j.ospino@usal.es
}

In this work we shall heavily rely on the formalism developed in [3], which is based on the $1+3$ formalism [4-7]; thus, even though we shall try to make this manuscript, as selfcontained as possible, we shall frequently refer the reader to [3], in order to avoid the rewriting of some of the equations.

In the next section we shall provide a summary of the main equations and concepts used in this study. Next we describe the null fluid outside the source. In order to stress the role of the vorticity in the emission of gravitational radiation we shall consider the vorticity-free case, which is shown to lead to either the static case or to the spherically symmetric case (Vaidya). The latter case is analyzed in some detail. A summary of the results is presented in the last section, and some intermediate equations are deployed in an appendix.

\section{Basic definitions and notation}

In this section we shall deploy all the variables required for our study, some details of the calculations are given in [3], and therefore we shall omit them here.

\subsection{The metric, the source, and the kinematical variables}

We shall consider, axially (and reflection) symmetric spacetimes. For such systems the line element may be written in "Weyl spherical coordinates" as

$\mathrm{d} s^{2}=-A^{2} \mathrm{~d} t^{2}+B^{2}\left(\mathrm{~d} r^{2}+r^{2} \mathrm{~d} \theta^{2}\right)+C^{2} \mathrm{~d} \phi^{2}+2 G \mathrm{~d} \theta \mathrm{d} t$,

where $A, B, C, G$ are positive functions of $t, r$, and $\theta$. We number the coordinates $x^{0}=t, x^{1}=r, x^{2}=\theta, x^{3}=\phi$.

We shall assume that our source is filled with an anisotropic and dissipative fluid, and is bounded by a time- 
like surface $\Sigma$, where junction (Darmois) conditions should be imposed.

The energy momentum tensor of the source may be written in the "canonical" form, as

$T_{\alpha \beta}=(\mu+P) V_{\alpha} V_{\beta}+P g_{\alpha \beta}+\Pi_{\alpha \beta}+q_{\alpha} V_{\beta}+q_{\beta} V_{\alpha}$.

In the above expression $V^{\mu}$ denotes the four-velocity assigned by certain observer, $\mu$ is the energy density (the eigenvalue of $T_{\alpha \beta}$ for eigenvector $V^{\alpha}$ ), $q_{\alpha}$ is the heat flux, whereas $P$ is the isotropic pressure, and $\Pi_{\alpha \beta}$ is the anisotropic tensor.

Since we choose the fluid to be comoving in our coordinates,

$V^{\alpha}=\left(\frac{1}{A}, 0,0,0\right) ; \quad V_{\alpha}=\left(-A, 0, \frac{G}{A}, 0\right)$.

Next, let us introduce the unit, spacelike vectors $\mathbf{K}, \mathbf{L}, \mathbf{S}$, with components

$K_{\alpha}=(0, B, 0,0) ; \quad L_{\alpha}=\left(0,0, \frac{\sqrt{A^{2} B^{2} r^{2}+G^{2}}}{A}, 0\right)$,

$L^{\alpha}=\left(\frac{G}{A \sqrt{A^{2} B^{2} r^{2}+G^{2}}}, 0, \frac{A}{\sqrt{A^{2} B^{2} r^{2}+G^{2}}}, 0\right)$,

$S_{\alpha}=(0,0,0, C)$,

satisfying the following relations:

$V_{\alpha} V^{\alpha}=-K^{\alpha} K_{\alpha}=-L^{\alpha} L_{\alpha}=-S^{\alpha} S_{\alpha}=-1$,

$V_{\alpha} K^{\alpha}=V^{\alpha} L_{\alpha}=V^{\alpha} S_{\alpha}=K^{\alpha} L_{\alpha}=K^{\alpha} S_{\alpha}=S^{\alpha} L_{\alpha}=0$.

For the energy density and the isotropic pressure, we have

$\mu=T_{\alpha \beta} V^{\alpha} V^{\beta}, \quad P=\frac{1}{3} h^{\alpha \beta} T_{\alpha \beta}$,

where

$h_{\beta}^{\alpha}=\delta_{\beta}^{\alpha}+V^{\alpha} V_{\beta}$,

whereas the anisotropic tensor may be expressed through three scalar functions defined by (see [3])

$\Pi_{K L}=K^{\alpha} L^{\beta} T_{\alpha \beta}$,

$\Pi_{I}=\left(2 K^{\alpha} K^{\beta}-L^{\alpha} L^{\beta}-S^{\alpha} S^{\beta}\right) T_{\alpha \beta}$,

$\Pi_{I I}=\left(2 L^{\alpha} L^{\beta}-S^{\alpha} S^{\beta}-K^{\alpha} K^{\beta}\right) T_{\alpha \beta}$.
Finally, we may write the heat flux vector in terms of two scalar functions:

$q_{\mu}=q_{I} K_{\mu}+q_{I I} L_{\mu}$,

or, in coordinate components (see [3])

$q^{\mu}=\left(\frac{q_{I I} G}{A \sqrt{A^{2} B^{2} r^{2}+G^{2}}}, \frac{q_{I}}{B}, \frac{A q_{I I}}{\sqrt{A^{2} B^{2} r^{2}+G^{2}}}, 0\right)$,

$q_{\mu}=\left(0, B q_{I}, \frac{\sqrt{A^{2} B^{2} r^{2}+G^{2}} q_{I I}}{A}, 0\right)$.

Of course, all the above quantities depend, in general, on $t, r, \theta$.

For the kinematical variables we obtain (see [3])

For the four acceleration

$a_{\alpha}=V^{\beta} V_{\alpha ; \beta}=a_{I} K_{\alpha}+a_{I I} L_{\alpha}$,

with

$a_{I}=\frac{A^{\prime}}{A B}$

$a_{I I}=\frac{A}{\sqrt{A^{2} B^{2} r^{2}+G^{2}}}\left[\frac{A, \theta}{A}+\frac{G}{A^{2}}\left(\frac{\dot{G}}{G}-\frac{\dot{A}}{A}\right)\right]$,

where the dot and the prime denote derivatives with respect to $t$ and $r$, respectively.

For the expansion scalar

$$
\begin{aligned}
\Theta= & V_{; \alpha}^{\alpha}=\frac{1}{A}\left(\frac{2 \dot{B}}{B}+\frac{\dot{C}}{C}\right) \\
& +\frac{G^{2}}{A\left(A^{2} B^{2} r^{2}+G^{2}\right)}\left(-\frac{\dot{A}}{A}-\frac{\dot{B}}{B}+\frac{\dot{G}}{G}\right) .
\end{aligned}
$$

Next, the shear tensor

$\sigma_{\alpha \beta}=V_{(\alpha ; \beta)}+a_{(\alpha} V_{\beta)}-\frac{1}{3} \Theta h_{\alpha \beta}$

may be defined through two scalar functions by

$$
\begin{aligned}
\sigma_{\alpha \beta}= & \frac{1}{3}\left(2 \sigma_{I}+\sigma_{I I}\right)\left(K_{\alpha} K_{\beta}-\frac{1}{3} h_{\alpha \beta}\right) \\
& +\frac{1}{3}\left(2 \sigma_{I I}+\sigma_{I}\right)\left(L_{\alpha} L_{\beta}-\frac{1}{3} h_{\alpha \beta}\right) .
\end{aligned}
$$

The above scalars may be written in terms of the metric functions and their derivatives as (see [3]) 


$$
\begin{aligned}
\sigma_{I}= & \frac{1}{A}\left(\frac{\dot{B}}{B}-\frac{\dot{C}}{C}\right) \\
& +\frac{G^{2}}{A\left(A^{2} B^{2} r^{2}+G^{2}\right)}\left(\frac{\dot{A}}{A}+\frac{\dot{B}}{B}-\frac{\dot{G}}{G}\right), \\
\sigma_{I I}= & \frac{1}{A}\left(\frac{\dot{B}}{B}-\frac{\dot{C}}{C}\right) \\
& +\frac{2 G^{2}}{A\left(A^{2} B^{2} r^{2}+G^{2}\right)}\left(-\frac{\dot{A}}{A}-\frac{\dot{B}}{B}+\frac{\dot{G}}{G}\right) .
\end{aligned}
$$

Finally, the vorticity may be described, either by the vorticity vector $\omega^{\alpha}$, or the vorticity tensor $\Omega^{\beta \mu}$, defined by

$\omega_{\alpha}=\frac{1}{2} \eta_{\alpha \beta \mu \nu} V^{\beta ; \mu} V^{\nu}=\frac{1}{2} \eta_{\alpha \beta \mu \nu} \Omega^{\beta \mu} V^{\nu}$,

where $\Omega_{\alpha \beta}=V_{[\alpha ; \beta]}+a_{[\alpha} V_{\beta]}$, and $\eta_{\alpha \beta \mu \nu}$ denote the LeviCivita tensor; we find a single component different from zero, producing

$\Omega_{\alpha \beta}=\Omega\left(L_{\alpha} K_{\beta}-L_{\beta} K_{\alpha}\right)$,

and

$\omega_{\alpha}=-\Omega S_{\alpha}$,

with the scalar function $\Omega$ given by

$\Omega=\frac{G\left(\frac{G^{\prime}}{G}-\frac{2 A^{\prime}}{A}\right)}{2 B \sqrt{A^{2} B^{2} r^{2}+G^{2}}}$.

2.2 The electric and magnetic part of the Weyl tensor and the super-Poynting vector

Let us now introduce the electric $\left(E_{\alpha \beta}\right)$ and magnetic $\left(H_{\alpha \beta}\right)$ parts of the Weyl tensor $\left(C_{\alpha \beta \gamma \delta}\right)$, defined as usual by

$$
\begin{aligned}
& E_{\alpha \beta}=C_{\alpha \nu \beta \delta} V^{\nu} V^{\delta}, \\
& H_{\alpha \beta}=\frac{1}{2} \eta_{\alpha \nu \epsilon \rho} C_{\beta \delta}{ }^{\epsilon \rho} V^{v} V^{\delta} .
\end{aligned}
$$

The electric part of the Weyl tensor has only three independent non-vanishing components, whereas only two components define the magnetic part. Thus we may write these tensors, in terms of three $\left(\mathcal{E}_{I}, \mathcal{E}_{I I}, \mathcal{E}_{K L}\right)$ and two $\left(H_{1}, H_{2}\right)$ scalar functions, respectively, as

$$
\begin{aligned}
E_{\alpha \beta}= & \frac{1}{3}\left(2 \mathcal{E}_{I}+\mathcal{E}_{I I}\right)\left(K_{\alpha} K_{\beta}-\frac{1}{3} h_{\alpha \beta}\right)+\frac{1}{3}\left(2 \mathcal{E}_{I I}+\mathcal{E}_{I}\right) \\
& \times\left(L_{\alpha} L_{\beta}-\frac{1}{3} h_{\alpha \beta}\right)+\mathcal{E}_{K L}\left(K_{\alpha} L_{\beta}+K_{\beta} L_{\alpha}\right)
\end{aligned}
$$

and

$$
H_{\alpha \beta}=H_{1}\left(S_{\alpha} K_{\beta}+S_{\beta} K_{\alpha}\right)+H_{2}\left(S_{\alpha} L_{\beta}+S_{\beta} L_{\alpha}\right) .
$$

Also, from the Riemann tensor we may define the three tensors $Y_{\alpha \beta}, X_{\alpha \beta}$, and $Z_{\alpha \beta}$ as

$Y_{\alpha \beta}=R_{\alpha \nu \beta \delta} V^{\nu} V^{\delta}$,

$X_{\alpha \beta}=\frac{1}{2} \eta_{\alpha \nu}{ }^{\epsilon \rho} R_{\epsilon \rho \beta \delta}^{\star} V^{\nu} V^{\delta}$,

and

$Z_{\alpha \beta}=\frac{1}{2} \epsilon_{\alpha \epsilon \rho} R_{\delta \beta}{ }^{\epsilon \rho} V^{\delta}$,

where $R_{\alpha \beta \nu \delta}^{\star}=\frac{1}{2} \eta_{\epsilon \rho \nu \delta} R_{\alpha \beta}{ }^{\epsilon \rho}$ and $\epsilon_{\alpha \beta \rho}=\eta_{\nu \alpha \beta \rho} V^{\nu}$.

The above tensors in turn may be decomposed, so that each of them is described through four scalar functions known as structure scalars [8]. These are (see [3] for details)

$Y_{T}=4 \pi(\mu+3 P), \quad X_{T}=8 \pi \mu$,

$Y_{I}=\mathcal{E}_{I}-4 \pi \Pi_{I}, \quad X_{I}=-\mathcal{E}_{I}-4 \pi \Pi_{I}$,

$Y_{I I}=\mathcal{E}_{I I}-4 \pi \Pi_{I I}, \quad X_{I I}=-\mathcal{E}_{I I}-4 \pi \Pi_{I I}$,

$Y_{K L}=\mathcal{E}_{K L}-4 \pi \Pi_{K L}, \quad X_{K L}=-\mathcal{E}_{K L}-4 \pi \Pi_{K L}$,

$Z_{I}=\left(H_{1}-4 \pi q_{I I}\right) ; \quad Z_{I I}=\left(H_{1}+4 \pi q_{I I}\right) ;$

$Z_{I I I}=\left(H_{2}-4 \pi q_{I}\right) ; \quad Z_{I V}=\left(H_{2}+4 \pi q_{I}\right)$.

From the above tensors, we may define the super-Poynting vector by

$P_{\alpha}=\epsilon_{\alpha \beta \gamma}\left(Y_{\delta}^{\gamma} Z^{\beta \delta}-X_{\delta}^{\gamma} Z^{\delta \beta}\right)$,

in our case, we may write

$P_{\alpha}=P_{I} K_{\alpha}+P_{I I} L_{\alpha}$,

with

$$
\begin{aligned}
P_{I}= & \frac{2 H_{2}}{3}\left(2 \mathcal{E}_{I I}+\mathcal{E}_{I}\right)+2 H_{1} \mathcal{E}_{K L} \\
& +\frac{32 \pi^{2} q_{I}}{3}\left[3(\mu+P)+\Pi_{I}\right]+32 \pi^{2} q_{I I} \Pi_{K L}, \\
P_{I I}= & -\frac{2 H_{1}}{3}\left(2 \mathcal{E}_{I}+\mathcal{E}_{I I}\right)-2 H_{2} \mathcal{E}_{K L} \\
& +\frac{32 \pi^{2} q_{I I}}{3}\left[3(\mu+P)+\Pi_{I I}\right]+32 \pi^{2} q_{I} \Pi_{K L} .
\end{aligned}
$$

In the theory of the super-Poynting vector, a state of gravitational radiation is associated to a non-vanishing component of the latter (see [9-12]). This is in agreement with the established link between the super-Poynting vector and the news functions [13], in the context of the Bondi-Sachs approach $[1,14]$.

We can identify two different contributions in (41). On the one hand we have contributions from the heat transport process. These are in principle independent of the magnetic 
part of the Weyl tensor, which explains why they remain in the spherically symmetric limit. Next we have contributions related to the gravitational radiation. These require, both the electric and the magnetic part of the Weyl tensor to be different from zero.

\section{The null fluid outside the source}

As mentioned in the Introduction, if the source produces gravitational radiation, then an entropy production factor should be present in its hydrodynamic description. This is so, because it has been discussed before in [1,2], gravitational radiation is an irreversible process (once causality condition is imposed), and therefore this fact should show up in the equation of state of the source. The obvious consequence of the presence of these dissipative processes within the source is the existence of incoherent radiation outside the source.

Thus, we assume that outside the source there is a null fluid, which due to the symmetry constraints has to be described by the energy momentum tensor of the form

$T_{\alpha \beta}=\lambda l_{\alpha} l_{\beta}+\epsilon n_{\alpha} n_{\beta}$,

where $\lambda$ and $\epsilon$ are two functions of $t, r, \theta$ related with the energy density of the null radiation in either direction $\mathbf{I}$ and $\mathbf{n}$, and these two null vectors are given by

$$
\begin{aligned}
l^{\alpha} & =\left(\frac{1}{A}, \frac{1}{B}, 0,0\right) \\
n^{\alpha} & =\left(\frac{1}{A}, 0,-\frac{G+\sqrt{A^{2} B^{2} r^{2}+G^{2}}}{A B^{2} r^{2}}, 0\right),
\end{aligned}
$$

or

$$
\begin{aligned}
l_{\alpha}= & \left(-A, B, \frac{G}{A}, 0\right), \\
n_{\alpha}= & {\left[-A-\frac{G}{A B^{2} r^{2}}\left(G+\sqrt{A^{2} B^{2} r^{2}+G^{2}}\right), 0,\right.} \\
& \left.-\frac{\sqrt{A^{2} B^{2} r^{2}+G^{2}}}{A}, 0\right] .
\end{aligned}
$$

We can now express the vectors $\mathbf{l}$ and $\mathbf{n}$ in terms of the tetrad vectors $\mathbf{V}, \mathbf{K}, \mathbf{L}, \mathbf{S}$.

Thus we find

$l_{\alpha}=V_{\alpha}+K_{\alpha}$,

and

$n_{\alpha}=\alpha V_{\alpha}+\gamma L_{\alpha}$, where

$\alpha \equiv 1+\frac{G}{A^{2} B^{2} r^{2}}\left(G+\sqrt{A^{2} B^{2} r^{2}+G^{2}}\right)$

and

$\gamma \equiv-1-\frac{\alpha G}{\sqrt{A^{2} B^{2} r^{2}+G^{2}}}$,

implying $\alpha=-\gamma$.

Now comparing (2) with (42), we find the following equivalence between different physical variables:

$$
\begin{gathered}
q_{I}=\lambda ; \quad q_{I I}=-\alpha^{2} \epsilon ; \quad P=\frac{1}{3}\left(\lambda+\epsilon \alpha^{2}\right) ; \quad \Pi_{K L}=0 ; \\
\Pi_{I}=2 \lambda-\epsilon \alpha^{2} ; \quad \Pi_{I I}=2 \epsilon \alpha^{2}-\lambda ; \quad \mu=\lambda+\epsilon \alpha^{2} .
\end{gathered}
$$

Observe that we have the equation of state $P=\frac{\mu}{3}$ corresponding to a pure radiation gas, as we should for a null fluid distribution.

Now, the interest of (50) resides in the fact that we can apply all the formalism developed in [3], to the study of our null fluid, just changing the variables according to the relationships indicated above. However, three important differences with the interior (source) case, must be pointed out, namely:

- Since we are now considering the outside of the source, the center of the fluid distribution is excluded from the space-time under study, accordingly no regular conditions at the center have to be imposed.

- Since our source is assumed to be bounded, we have to impose asymptotic conditions at spatial infinity. In particular we shall assume that our line element approaches asymptotically the Minkowski metric.

- At the boundary of the source, appropriate junction conditions (Darmois) must be imposed to avoid the presence of shells. Even though, for the study presented here, such conditions will not be used explicitly, they have to be taken into account for any specific global model, describing the space-time outside and inside the fluid distribution.

As should be obvious, there is not a unique space-time corresponding to our null fluid distribution (as is the case for the spherically symmetric situation), we have instead an infinite number of possible solutions. Thus our main purpose here is not to provide specific solutions to this case, but rather to reveal some specific aspects of the problem. More precisely, we would like to exhibit the role played by the vorticity in the properties of the null fluid. Thus we shall consider the vorticity-free case. 
3.1 The case without vorticity $G=0$

Thus, let us assume the vanishing of the vorticity $(G=0)$ then $\alpha=-\gamma=1$, which implies because of (50)

$$
\begin{gathered}
q_{I}=\lambda ; \quad q_{I I}=-\epsilon ; \quad P=\frac{1}{3}(\lambda+\epsilon) ; \quad \Pi_{K L}=0 ; \\
\Pi_{I}=2 \lambda-\epsilon ; \quad \Pi_{I I}=2 \epsilon-\lambda ; \quad \mu=\lambda+\epsilon .
\end{gathered}
$$

Also in this case

$$
\begin{aligned}
\sigma_{I} & =\sigma_{I I}=\bar{\sigma}=\frac{1}{A}\left(\frac{\dot{B}}{B}-\frac{\dot{C}}{C}\right), \\
\Theta & =\frac{1}{A}\left(\frac{2 \dot{B}}{B}+\frac{\dot{C}}{C}\right) .
\end{aligned}
$$

First, let us recall that as $r \rightarrow \infty$ we must recover the Minkowski space-time, implying that we can write, at least sufficiently far from the source,

$$
\begin{aligned}
& A(t, r, \theta)=\sum_{n \geq 0} \frac{A^{(n)}(t, \theta)}{r^{n}} ; \quad B(t, r, \theta)=\sum_{n \geq 0} \frac{B^{(n)}(t, \theta)}{r^{n}} ; \\
& C(t, r, \theta)=\sum_{n \geq-1} \frac{C^{(n)}(t, \theta)}{r^{n}},
\end{aligned}
$$

where $A^{(0)}=B^{(0)}=1, C^{(-1)}=\sin \theta, C^{(0)}=0$.

Also we can write

$\lambda(t, r, \theta)=\sum_{n \geq 1} \frac{\lambda^{(n)}(t, \theta)}{r^{n}} ; \quad \epsilon(t, r, \theta)=\sum_{n \geq 1} \frac{\epsilon^{(n)}(t, \theta)}{r^{n}}$.

We shall first prove that in the case $G=0$, either $\lambda=\epsilon=0$ or we have the spherically symmetric situation.

Indeed, using (53) and (54) in (A8), (A9), and (A15), we obtain at order $O\left(r^{-1}\right)$ and $O\left(r^{-3}\right)$

$\epsilon^{(1)}=\epsilon^{(2)}=0$.

Next, combining (A14) with (A15) we may write

$$
\begin{aligned}
& -\frac{\dot{\epsilon}}{A}+\frac{\epsilon, \theta}{B r}+\frac{\epsilon}{B}\left(\frac{A^{\prime}}{A}-\frac{(B r)^{\prime}}{B r}\right) \\
& +\frac{\epsilon}{B r}\left(\frac{2 A_{, \theta}}{A}+\frac{B, \theta}{B}+\frac{C_{\theta}}{C}\right)-\frac{\epsilon}{A}\left(\frac{3 \dot{B}}{B}+\frac{\dot{C}}{C}\right)=0 .
\end{aligned}
$$

Feeding back (53) and (54) into (56) and using (80), we find at order $O\left(r^{-3}\right)$ and $O\left(r^{-4}\right)$ that

$\dot{\epsilon}^{(3)}=0, \quad \dot{\epsilon}^{(4)}=\epsilon_{, \theta}^{(3)}+\epsilon^{(3)}\left(\cot \theta-1-3 \dot{B}^{(1)}\right)$.

Now, for any physically meaningful radiating process, we must demand $\epsilon$ to vanish out of a finite time interval, implying that $\dot{\epsilon}^{(3)}=0 \Rightarrow \epsilon^{(3)}=0$, which in turn, using (57), implies $\dot{\epsilon}^{(4)}=0 \Rightarrow \epsilon^{(4)}=0$.

Following this line of arguments at all possible orders, it is found (the Mathematica package was used for this purpose) that

$\dot{\epsilon}^{(n)}=0, \forall n \Rightarrow \epsilon^{(n)}=0 \Rightarrow \epsilon=0$.

Then from (A15) we see that either $\lambda=0$, or we have a spherically symmetric system with $A_{, \theta}=B_{, \theta}=0$.

Let us first consider the case $\lambda=\epsilon=0$. Then we obtain from (A8) and (A9)

$\bar{\sigma}^{\prime} C_{, \theta}-\bar{\sigma}_{, \theta} C^{\prime}=0$

and

$\Theta^{\prime} C_{, \theta}-\Theta_{, \theta} C^{\prime}=0$,

whose combination produces, using (52),

$\left(\frac{\dot{C}}{A C}\right)^{\prime} C_{, \theta}-\left(\frac{\dot{C}}{A C}\right)_{, \theta} C^{\prime}=0$.

Feeding back (53) into the above equation, it is easy to prove that for any $n \geq 1$

$\dot{C}^{(n)}=\frac{f(t)}{\sin ^{n} \theta}$,

where $f$ is an arbitrary function of its argument.

Then from the regularity conditions on the symmetry axis we must put $f=0$, which implies

$\dot{C}=0$.

The above result implies, because of (52),

$\Theta=2 \bar{\sigma}$.

Then integration of (A8), using the above equation and (59), produces

$\bar{\sigma}=g(t) C$,

where $g$ is an arbitrary function of its argument.

Finally, feeding back the above expression into (A10) and (A11) we obtain

$H_{1}=-\frac{g(t) C_{, \theta}}{B r} ; \quad H_{2}=\frac{g(t) C^{\prime}}{B}$.

However, since we must impose the asymptotic condition $H_{1}, H_{2} \Rightarrow 0$ as $r \rightarrow \infty$, we must put $g(t)=0$, implying 
that the metric is static. We shall further discuss this issue in the last section.

Next we shall consider the spherically symmetric case.

\subsection{The spherically symmetric limit}

It is instructive to reproduce the spherically symmetric case (Vaidya), in the context of the formalism considered here.

In the spherically symmetric case the following conditions apply:

$H_{1}=H_{2}=\bar{\sigma}=a_{I I}=q_{I I}=\epsilon=\Pi_{K L}=A_{, \theta}=B_{, \theta}=0$

and

$C=B r \sin \theta ; \quad q_{I}=\lambda ; \quad a_{I}=\frac{A^{\prime}}{A B}$,

implying

$\Theta=\frac{3 \dot{B}}{A B} ; \quad \Pi_{I}=2 \lambda ; \quad \Pi_{I I}=-\lambda ; \quad \mu=\lambda$,

$P=\frac{\lambda}{3} ; \quad 2 \Pi_{I I}+\Pi_{I}=\mathcal{E}_{K L}=Y_{K L}=X_{K L}=0$.

Using the conditions above in (A8) we obtain

$\left(\frac{1}{A} \frac{\dot{B}}{B}\right)^{\prime}=4 \pi B \lambda$

whereas (A2) and (A4) produce, respectively,

$2 a_{I}^{\prime}+a_{I}\left(\frac{2 A^{\prime}}{A}-\frac{2(B r)^{\prime}}{B r}\right)-B \mathcal{E}_{I}+8 \pi B \lambda=0$,

$a_{I}^{\prime}+a_{I}\left(\frac{A^{\prime}}{A}-\frac{(B r)^{\prime}}{B r}\right)+B \mathcal{E}_{I I}+4 \pi B \lambda=0$.

From the two equations above, it follows at once that

$2 \mathcal{E}_{I I}+\mathcal{E}_{I}=0 \Rightarrow 2 X_{I I}+X_{I}=2 Y_{I I}+Y_{I}=0$.

Next, (A1) reads

$\frac{1}{A} \dot{\Theta}+\frac{1}{3} \Theta^{2}-\frac{1}{B}\left[a_{I}^{\prime}+a_{I}\left(\frac{A^{\prime}}{A}+\frac{2(B r)^{\prime}}{B r}\right)\right]+8 \pi \lambda=0$,

whereas Eqs. (A3), (A6), (A9), (A10), and (A11) become identities.

Let us now turn to the conservation laws. We obtain from (A12)

$\frac{\dot{\lambda}}{A}+\frac{4 \lambda \dot{B}}{A B}+\frac{1}{B}\left[\lambda^{\prime}+2 \lambda\left(\frac{A^{\prime}}{A}+\frac{(B r)^{\prime}}{B r}\right)\right]=0$, whereas (A13) produces the same result as (75), and (A14) and (A15) become identities.

Next, we obtain from (A16), (A18), (A19), and (A20), respectively,

$\frac{\dot{\mathcal{E}}_{I}}{3 A}+\frac{4 \pi \dot{\lambda}}{A}+\frac{\mathcal{E}_{I} \Theta}{3}=-\frac{8 \pi}{3} \lambda \Theta-\frac{4 \pi}{B}\left(\lambda^{\prime}+\frac{2 A^{\prime} \lambda}{A}\right)$,

$\frac{\dot{\mathcal{E}}_{I I}}{3 A}+\frac{\mathcal{E}_{I I} \Theta}{3}=-\frac{4 \pi}{3} \lambda \Theta-\frac{4 \pi \lambda(B r)^{\prime}}{B^{2} r}$,

$-\frac{\dot{\mathcal{E}}_{I}+\dot{\mathcal{E}}_{I I}}{3 A}-\frac{\left(\mathcal{E}_{I}+\mathcal{E}_{I I}\right) \Theta}{3}=-\frac{4 \pi}{3} \lambda \Theta-\frac{4 \pi \lambda}{B} \frac{(B r)^{\prime}}{B r}$,

$\frac{1}{3 B}\left[\mathcal{E}_{I}^{\prime}+3 \mathcal{E}_{I} \frac{(B r)^{\prime}}{B r}\right]+8 \pi \lambda \frac{(B r)^{\prime}}{B^{2} r}=-\frac{8 \pi \lambda}{3} \Theta$,

whereas (A17), (A21), (A22), and (A23) become identities.

Also, it is a simple matter to check that (A24) becomes identical to (79), and that (77) and (78) are equivalent.

We can now determine, asymptotically, the space-time (Vaidya) by the iterative procedure sketched below.

First, let us notice that Eqs. (70), (74), and (75) may be written, respectively, as

$\frac{\dot{B}^{\prime}}{A B}-\frac{A^{\prime} \dot{B}}{A^{2} B}-\frac{\dot{B} B^{\prime}}{A B^{2}}=4 \pi B \lambda$,

$\frac{3 \ddot{B}}{A^{2} B}-\frac{3 \dot{A} \dot{B}}{A^{3} B}-\frac{A^{\prime \prime}}{A B^{2}}-\frac{A^{\prime} B^{\prime}}{A B^{3}}-\frac{2 A^{\prime}}{A B^{2} r}=-8 \pi \lambda$,

$\frac{\dot{\lambda}}{A}+\frac{4 \lambda \dot{B}}{A B}+\frac{\lambda^{\prime}}{B}+\frac{2 A^{\prime} \lambda}{A B}+\frac{2 \lambda B^{\prime}}{B^{2}}+\frac{2 \lambda}{B r}=0$.

Using (53), and evaluating (80) at the order $O\left(r^{-1}\right)$, $O\left(r^{-2}\right)$, and $O\left(r^{-3}\right)$, and (82) at order $O\left(r^{-3}\right)$, we find that (obviously in the spherically symmetric case the coefficients $A^{(n)}, B^{(n)}$, and $\lambda^{(n)}$, do not depend on $\theta$ )

$\lambda^{(1)}=0 ; \quad \lambda^{(2)}=-\frac{\dot{B}^{(1)}}{4 \pi} ; \quad \dot{\lambda}^{(3)}=-4 \lambda^{(2)} \dot{B}^{(1)}$.

Next, evaluating (82) at order $O\left(r^{-2}\right)$, it follows that

$\dot{\lambda}^{(2)}=0 \Rightarrow \lambda^{(2)}=$ constant.

This last result together with (83) produces

$B^{(1)}=-4 \pi \lambda^{(2)} t+$ constant

and

$\lambda^{(3)}=16 \pi\left(\lambda^{(2)}\right)^{2} t+$ constant.

Next, the order $O\left(r^{-3}\right)$ in (80) produces

$-2 \dot{B}^{(2)}=12 \pi B^{(1)} \lambda^{(2)}+8 \pi A^{(1)} \lambda^{(2)}+4 \pi \lambda^{(3)}$. 
From the above equation we cannot obtain the time dependence of $B^{(2)}$ since we do not know $A^{(1)}$.

So, let us turn to $(81)$; at the highest order $\left(O\left(r^{-1}\right)\right)$ we find

$\ddot{B}^{(1)}=0$,

a well-known result, whereas from the order $O\left(r^{-2}\right)$ it follows

$12 \pi \lambda^{(2)} \dot{A}^{(1)}+3 \ddot{B}^{(2)}=-8 \pi \lambda^{(2)}$.

Now, taking the $t$-derivative of (87), solving for $\ddot{B}^{(2)}$ and feeding back into (89) we obtain

$\lambda^{(2)}=\frac{1}{3 \pi}$.

In other words we still need a function of time in order to determine the time dependence of $A^{(1)}$ and $B^{(2)}$.

So, let us look for the next orders in (80), (81), and (82). Thus, from the order $O\left(r^{-4}\right)$ in (80), $O\left(r^{-3}\right)$ in (81) and $O\left(r^{-4}\right)$ in (82) we obtain, respectively,

$$
\begin{aligned}
4 \pi \lambda^{(4)}= & -\dot{B}^{(1)}\left[3\left(A^{(1)}\right)^{2}+6\left(B^{(1)}\right)^{2}+5 A^{(1)} B^{(1)}-3 A^{(2)}\right. \\
& \left.-4 B^{(2)}\right]+\dot{B}^{(2)}\left(5 B^{(1)}+3 A^{(1)}\right)-3 \dot{B}^{(3)}, \quad(91) \\
-8 \pi \lambda^{(3)}= & -3 \dot{B}^{(2)} \dot{A}^{(1)}+\dot{B}^{(1)}\left[-3 \dot{A}^{(2)}+3 \dot{A}^{(1)} B^{(1)}\right. \\
& \left.+9 \dot{A}^{(1)} A^{(1)}\right]+3 \ddot{B}^{(3)}-3 \ddot{B}^{(2)}\left(2 A^{(1)}+B^{(1)}\right),
\end{aligned}
$$

$$
\begin{aligned}
\dot{\lambda}^{(4)}-\dot{\lambda}^{(3)} A^{(1)}= & \lambda^{(2)}\left(4 \dot{B}^{(1)} A^{(1)}+4 \dot{B}^{(1)} B^{(1)}-4 \dot{B}^{(2)}\right. \\
& \left.+2 A^{(1)}+2 B^{(1)}\right)+\lambda^{(3)}\left(1-4 \dot{B}^{(1)}\right) .
\end{aligned}
$$

Once $B^{(2)}$ or $A^{(1)}$ have been determined, we can find $\lambda^{(4)}$ from (93).

Next, taking the time derivative of (91) and combining with (92), we obtain

$$
\begin{aligned}
4 \pi \dot{\lambda}^{(4)}-8 \pi \lambda^{(3)}= & -\dot{B}^{(1)}\left(-3 A^{(1)} \dot{A}^{(1)}+12 B^{(1)} \dot{B}^{(1)}\right. \\
& \left.+2 B^{(1)} \dot{A}^{(1)}+5 A^{(1)} \dot{B}^{(1)}-9 \dot{B}^{(2)}\right) \\
& +\ddot{B}^{(2)}\left(2 B^{(1)}-3 A^{(1)}\right) .
\end{aligned}
$$

Thus we have no further information as regards the time dependence of $A^{(2)}$ or $B^{(3)}$, which implies that we have to provide the time dependence of either one of them. Following this procedure ad nauseam we see, as expected, that the metric is obtained up to an arbitrary function of $t$ and $r$. Thus, whereas the Vaidya metric has an extremely simple form in null coordinates, in the present approach it is only possible to construct it asymptotically, as a series expansion.

\section{Conclusions}

We have tackled the problem of describing the outer spacetime of axially symmetric sources of gravitational radiation, based on the Bondi conjecture [1] (confirmed in [2]), according to which the process of gravitational radiation is an irreversible one, and therefore must entail dissipative processes within the source.

The ensuing consequence of this is that there should be incoherent radiation (null fluid) at the outside of the source, produced by those dissipative processes. Keeping this fact in mind, we should remark that the Bondi-Sachs metric [1], [14], should be regarded as an approximation to the spacetime outside the source, when the null fluid produced by the dissipative processes is neglected.

Starting with the description of this null fluid, we apply the formalism developed in [3] to study some of the properties of such a null fluid.

As the main result of our study we find that the absence of vorticity implies that the exterior space-time is either static or spherically symmetric (Vaidya). Reinforcing thereby the fundamental role of vorticity in any process involving production of gravitational radiation, already stressed in [15], [16].

There exists still the possibility of the non-radiative, nonstatic solutions considered by Bondi in [1] (see also [17]), which corresponds to the case $G \neq 0, H_{1}=H_{2}=0$. Indeed, in this case the Bondi news function vanishes [17], and therefore the system does not radiate gravitational waves (this is also evident from (41)), even though it may be time dependent. In particular, it can be shown that the mass, the "dipole" and the "quadrupole" moments (as defined in [1]) correspond to a static situation. However, the time dependence might enter through coefficients of higher order in the metric, giving rise to what Bondi calls "non-natural, nonradiative moving systems". In the latter case the three first moments are time independent, but the system allows for a time dependence of the higher moments (see also [18,19]). As unlikely as this situation may be from the physical point of view, it cannot be ruled out.

It must be kept in mind that throughout our discussion we have restricted ourselves to physically meaningful situations, where the source is bounded and the radiation process takes place during a finite time interval. Obviously if we relax either of these conditions, other cases might appear, even though they would be devoid of physical relevance.

Finally, we have indicated how to recover (at least asymptotically) the Vaidya metric. Unlike the null coordinates, our 
coordinates do not allow for a simple expression for the corresponding line element.

Acknowledgements $\mathrm{LH}$ and JO acknowledge financial support from the Spanish Ministry of Science and Innovation (Grant FIS2009-07238) and Fondo Europeo de Desarrollo Regional (FEDER) (Grant FIS201565140-P) (MINECO/FEDER).

Open Access This article is distributed under the terms of the Creative Commons Attribution 4.0 International License (http://creativecomm ons.org/licenses/by/4.0/), which permits unrestricted use, distribution, and reproduction in any medium, provided you give appropriate credit to the original author(s) and the source, provide a link to the Creative Commons license, and indicate if changes were made.

Funded by SCOAP ${ }^{3}$.

\section{Appendix A: Some basic equations}

In the following, we shall present the main equations of the formalism, specialized for the case with $G=0$ (which of course includes as a particular case the spherically symmetric situation).

Thus, from B1, B2, B3, and B4 in [3] we get, respectively,

$$
\begin{array}{r}
\frac{1}{A} \dot{\Theta}+\frac{1}{3} \Theta^{2}+\frac{2}{3} \bar{\sigma}^{2}-\frac{1}{B}\left[a_{I}^{\prime}+a_{I}\left(\frac{A^{\prime}}{A}+\frac{(B r)^{\prime}}{B r}+\frac{C^{\prime}}{C}\right)\right] \\
-\frac{1}{B r}\left[a_{I I, \theta}+a_{I I}\left(\frac{A_{, \theta}}{A}+\frac{B, \theta}{B}+\frac{C_{, \theta}}{C}\right)\right]+8 \pi(\lambda+\epsilon)=0,
\end{array}
$$$$
\frac{1}{A} \dot{\bar{\sigma}}+\frac{1}{A^{2}}\left[\left(\frac{\dot{B}}{B}\right)^{2}-\left(\frac{\dot{C}}{C}\right)^{2}\right]-\frac{1}{B}\left[2 a_{I}^{\prime}\right.
$$$$
\left.+a_{I}\left(\frac{2 A^{\prime}}{A}-\frac{(B r)^{\prime}}{B r}-\frac{C^{\prime}}{C}\right)\right]+\frac{1}{B r}\left[a_{I I, \theta}\right.
$$$$
\left.+a_{I I}\left(\frac{A_{, \theta}}{A}-\frac{2 B_{, \theta}}{B}+\frac{C_{, \theta}}{C}\right)\right]
$$$$
+\mathcal{E}_{I}-4 \pi(2 \lambda-\epsilon)=0,
$$

$$
\begin{aligned}
& \frac{1}{B}\left[a_{I I}^{\prime}-a_{I I} \frac{(B r)^{\prime}}{B r}\right]+\frac{1}{B r}\left[a_{I, \theta}+a_{I}\left(\frac{2 A_{, \theta}}{A}-\frac{B, \theta}{B}\right)\right] \\
& \quad-2 \mathcal{E}_{K L}=0,
\end{aligned}
$$

$$
\begin{aligned}
& \frac{1}{A} \dot{\bar{\sigma}}+\frac{1}{A^{2}}\left[\left(\frac{\dot{B}}{B}\right)^{2}-\left(\frac{\dot{C}}{C}\right)^{2}\right] \\
& +\frac{1}{B}\left[a_{I}^{\prime}+a_{I}\left(\frac{A^{\prime}}{A}-\frac{2(B r)^{\prime}}{B r}+\frac{C^{\prime}}{C}\right)\right] \\
& \quad-\frac{1}{B r}\left[2 a_{I I, \theta}+a_{I I}\left(\frac{2 A_{, \theta}}{A}-\frac{B_{, \theta}}{B}-\frac{C_{, \theta}}{C}\right)\right] \\
& +\mathcal{E}_{I I}-4 \pi(2 \epsilon-\lambda)=0 .
\end{aligned}
$$

From (A2) and (A4) we obtain

$$
\begin{aligned}
\frac{1}{B} & {\left[-a_{I}^{\prime}+a_{I}\left(-\frac{A^{\prime}}{A}+\frac{(B r)^{\prime}}{B r}\right)\right] } \\
& +\frac{1}{B r}\left[a_{I I, \theta}+a_{I I}\left(\frac{A_{, \theta}}{A}-\frac{B_{, \theta}}{B}\right)\right] \\
= & \frac{\mathcal{E}_{I I}-\mathcal{E}_{I}}{3}-4 \pi(\epsilon-\lambda) .
\end{aligned}
$$

Next, from B5 in [3], we obtain

$$
-\frac{1}{B}\left[a_{I I}^{\prime}+a_{I I} \frac{(B r)^{\prime}}{B r}\right]+\frac{1}{B r}\left[a_{I, \theta}+a_{I} \frac{B, \theta}{B}\right]=0,
$$

which combined with (A3) produces

$\mathcal{E}_{K L}=\frac{1}{B^{2} r}\left[\frac{A_{, \theta}^{\prime}}{A}-\frac{A^{\prime} B_{, \theta}}{A B}-\frac{A_{, \theta}(B r)^{\prime}}{A B r}\right]$.

Next, from B6, B7, B8, and B9 in [3] we get, respectively,

$$
\begin{aligned}
& \frac{1}{3}(2 \Theta-\bar{\sigma})^{\prime}-\bar{\sigma} \frac{C^{\prime}}{C}=8 \pi B \lambda, \\
& \frac{1}{3}(2 \Theta-\bar{\sigma})_{, \theta}-\bar{\sigma} \frac{C_{, \theta}}{C}=-8 \pi B r \epsilon, \\
& H_{1}=-\frac{1}{2 B r}\left(\bar{\sigma}_{, \theta}+\bar{\sigma} \frac{C_{, \theta}}{C}\right), \\
& H_{2}=\frac{1}{2 B}\left(\bar{\sigma}^{\prime}+\bar{\sigma} \frac{C^{\prime}}{C}\right) .
\end{aligned}
$$

We have next the conservation laws (Eqs. A6, A7 in [3]), which read

$$
\begin{aligned}
& \frac{1}{A}(\dot{\lambda}+\dot{\epsilon})+\frac{(\lambda+\epsilon)}{A}\left(\frac{3 \dot{B}}{B}+\frac{\dot{C}}{C}\right) \\
& +\frac{1}{B}\left[\lambda^{\prime}+\lambda\left(\frac{2 A^{\prime}}{A}+\frac{(B r)^{\prime}}{B r}+\frac{C^{\prime}}{C}\right)\right] \\
& -\frac{1}{B r}\left[\epsilon, \theta+\epsilon\left(\frac{2 A_{, \theta}}{A}+\frac{B, \theta}{B}+\frac{C_{, \theta}}{C}\right)\right]=0, \\
& \frac{\dot{\lambda}}{A}+\frac{\lambda^{\prime}}{B}+\frac{\lambda}{B}\left[\frac{2 A^{\prime}}{A}+\frac{(B r)^{\prime}}{B r}+\frac{C^{\prime}}{C}\right]+\frac{\epsilon}{B}\left[\frac{A^{\prime}}{A}-\frac{(B r)^{\prime}}{B r}\right] \\
& +\frac{\lambda}{A}\left(\frac{3 \dot{B}}{B}+\frac{\dot{C}}{C}\right)=0,
\end{aligned}
$$

and

$$
\begin{aligned}
& -\frac{\dot{\epsilon}}{A}+\frac{\epsilon, \theta}{B r}+\frac{\lambda}{B r}\left(\frac{A_{, \theta}}{A}-\frac{B_{, \theta}}{B}\right)+\frac{\epsilon}{B r}\left(\frac{2 A_{, \theta}}{A}+\frac{B_{, \theta}}{B}+\frac{C_{\theta}}{C}\right) \\
& -\frac{\epsilon}{A}\left(\frac{3 \dot{B}}{B}+\frac{\dot{C}}{C}\right)=0 ;
\end{aligned}
$$

the combination of the last three equations produces

$$
\frac{\lambda}{B r}\left(\frac{A_{, \theta}}{A}-\frac{B, \theta}{B}\right)=\frac{\epsilon}{B}\left[\frac{A^{\prime}}{A}-\frac{(B r)^{\prime}}{B r}\right] .
$$




$$
\begin{aligned}
& \frac{\dot{\mathcal{E}}_{I}}{3 A}+\frac{4 \pi \dot{\lambda}}{A}+\frac{\mathcal{E}_{I} \Theta}{3}+\frac{\mathcal{E}_{I I} \bar{\sigma}}{3}-\frac{1}{B r}\left[H_{1, \theta}+H_{1}\left(\frac{2 A, \theta}{A}+\frac{C_{, \theta}}{C}\right)\right] \\
& +\frac{H_{2}}{B}\left[\frac{(B r)^{\prime}}{B r}-\frac{C^{\prime}}{C}\right]=-\frac{4 \pi}{3}(2 \lambda+\epsilon)(\bar{\sigma}+\theta) \\
& -\frac{4 \pi}{B}\left(\lambda^{\prime}+\frac{2 A^{\prime} \lambda}{A}\right)+\frac{4 \pi \epsilon B_{, \theta}}{B^{2} r}, \\
& \frac{\dot{\mathcal{E}}_{K L}}{A}+\mathcal{E}_{K L}(\Theta-\bar{\sigma})+\frac{1}{2 B}\left[H_{1}^{\prime}+H_{1}\left(\frac{2 A^{\prime}}{A}-\frac{(B r)^{\prime}}{B r}+\frac{2 C^{\prime}}{C}\right)\right] \\
& -\frac{1}{2 B r}\left[H_{2, \theta}+H_{2}\left(\frac{2 A_{, \theta}}{A}-\frac{B_{, \theta}}{B}+\frac{2 C_{, \theta}}{C}\right)\right] \\
& =\frac{2 \pi}{B}\left[\epsilon^{\prime}+\epsilon\left(\frac{2 A^{\prime}}{A}-\frac{(B r)^{\prime}}{B r}\right)\right] \\
& -\frac{2 \pi}{B r}\left[\lambda_{, \theta}+\lambda\left(\frac{2 A_{, \theta}}{A}-\frac{B_{, \theta}}{B}\right)\right] \text {, } \\
& \frac{\dot{\mathcal{E}}_{I I}}{3 A}+\frac{4 \pi \dot{\epsilon}}{A}+\frac{\mathcal{E}_{I I} \Theta}{3}+\frac{\mathcal{E}_{I} \bar{\sigma}}{3}+\frac{1}{B}\left[H_{2}^{\prime}+H_{2}\left(\frac{2 A^{\prime}}{A}+\frac{C^{\prime}}{C}\right)\right] \\
& \frac{1}{B}\left[H_{1}^{\prime}+H_{1}\left(\frac{(B r)^{\prime}}{B r}+\frac{2 C^{\prime}}{C}\right)\right]+\frac{1}{B r}\left[H_{2, \theta}\right. \\
& \left.+H_{2}\left(\frac{B_{, \theta}}{B}+\frac{2 C_{, \theta}}{C}\right)\right]=\frac{4 \pi}{B}\left[\epsilon^{\prime}+\frac{\epsilon(B r)^{\prime}}{B r}\right] \\
& +\frac{4 \pi}{B r}\left(\lambda_{, \theta}+\frac{\lambda B_{, \theta}}{B}\right) \text {, } \\
& -\frac{1}{3 B r}\left[\mathcal{E}_{I, \theta}+\mathcal{E}_{I}\left(\frac{2 A_{, \theta}}{A}+\frac{C_{, \theta}}{C}\right)\right]-\frac{1}{3 B r}\left[\mathcal{E}_{I I, \theta}\right. \\
& \left.+\mathcal{E}_{I I}\left(\frac{A_{, \theta}}{A}+\frac{2 C_{, \theta}}{C}\right)\right]+\frac{\mathcal{E}_{K L}}{B}\left(\frac{A^{\prime}}{A}-\frac{C^{\prime}}{C}\right) \\
& +\frac{4 \pi}{B r}\left(\lambda, \theta+\frac{\lambda B_{, \theta}}{B}\right) \\
& -\frac{4 \pi \epsilon}{B r} \frac{B, \theta}{B}+\frac{\dot{H}_{1}}{A}+H_{1} \Theta+\frac{4 \pi \epsilon}{3}(\Theta+\bar{\sigma})=0, \\
& \frac{1}{3 B}\left[\mathcal{E}_{I}^{\prime}+\mathcal{E}_{I}\left(\frac{A^{\prime}}{A}+\frac{2 C^{\prime}}{C}\right)\right]+\frac{1}{3 B}\left[\mathcal{E}_{I I}^{\prime}\right. \\
& \left.+\mathcal{E}_{I I}\left(\frac{2 A^{\prime}}{A}+\frac{C^{\prime}}{C}\right)\right]-\frac{\mathcal{E}_{K L}}{B r}\left(\frac{A_{, \theta}}{A}-\frac{C_{, \theta}}{C}\right) \\
& -\frac{4 \pi}{B}\left[\epsilon^{\prime}+\frac{\epsilon(B r)^{\prime}}{B r}\right]+\frac{4 \pi \lambda}{B} \frac{(B r)^{\prime}}{B r} \\
& +\frac{\dot{H}_{2}}{A}+H_{2} \Theta+\frac{4 \pi \lambda}{3}(\Theta+\bar{\sigma})=0 \text {. }
\end{aligned}
$$$$
-\frac{H_{1}}{B r}\left(\frac{B_{, \theta}}{B}-\frac{C_{, \theta}}{C}\right)=-\frac{4 \pi}{3}(\lambda+2 \epsilon)(\bar{\sigma}+\theta)
$$$$
+\frac{4 \pi}{B r}\left(\epsilon_{, \theta}+\frac{2 A_{, \theta} \epsilon}{A}\right)-\frac{4 \pi \lambda(B r)^{\prime}}{B^{2} r},
$$

$$
\begin{aligned}
& -\frac{\dot{\mathcal{E}}_{I}+\dot{\mathcal{E}}_{I I}}{3 A}-\frac{\left(\mathcal{E}_{I}+\mathcal{E}_{I I}\right)(\Theta+\bar{\sigma})}{3}+\frac{1}{B r}\left[H_{1, \theta}\right. \\
& \left.+H_{1}\left(\frac{2 A, \theta}{A}+\frac{B_{, \theta}}{B}\right)\right]-\frac{1}{B}\left[H_{2}^{\prime}+H_{2}\left(\frac{2 A^{\prime}}{A}+\frac{(B r)^{\prime}}{B r}\right)\right] \\
& =\frac{4 \pi}{3}(\lambda+\epsilon)(2 \bar{\sigma}-\Theta)-\frac{4 \pi \lambda}{B} \frac{C^{\prime}}{C}+\frac{4 \pi \epsilon C_{, \theta}}{B C r},
\end{aligned}
$$$$
\frac{1}{3 B}\left[\mathcal{E}_{I}^{\prime}+\mathcal{E}_{I}\left(\frac{(B r)^{\prime}}{B r}+\frac{2 C^{\prime}}{C}\right)\right]-\frac{\mathcal{E}_{I I}}{3 B}\left[\frac{(B r)^{\prime}}{B r}-\frac{C^{\prime}}{C}\right]
$$$$
+\frac{1}{B r}\left[\mathcal{E}_{K L, \theta}+\mathcal{E}_{K L}\left(\frac{2 B, \theta}{B}+\frac{C_{, \theta}}{C}\right)\right]-\frac{4 \pi}{B}\left[\epsilon^{\prime}+\frac{\epsilon(B r)^{\prime}}{B r}\right]
$$$$
+\frac{4 \pi \lambda}{B}\left[\frac{(B r)^{\prime}}{B r}+\frac{C^{\prime}}{C}\right]-H_{2} \bar{\sigma}=-\frac{4 \pi \lambda}{3}(2 \Theta-\bar{\sigma}),
$$

$\frac{1}{3 B r}\left[\mathcal{E}_{I I, \theta}+\mathcal{E}_{I I}\left(\frac{B_{, \theta}}{B}+\frac{2 C_{, \theta}}{C}\right)\right]-\frac{\mathcal{E}_{I}}{3 B r}\left(\frac{B, \theta}{B}-\frac{C_{, \theta}}{C}\right)$$$
+\frac{1}{B}\left[\mathcal{E}_{K L}^{\prime}+\mathcal{E}_{K L}\left(\frac{2(B r)^{\prime}}{B r}+\frac{C^{\prime}}{C}\right)\right]-\frac{4 \pi}{B r}\left(\lambda_{, \theta}+\frac{\lambda B_{, \theta}}{B}\right)
$$$$
+\frac{4 \pi \epsilon}{B r}\left(\frac{B, \theta}{B}+\frac{C, \theta}{C}\right)+H_{1} \bar{\sigma}=\frac{4 \pi \epsilon}{3}(2 \Theta-\bar{\sigma}),
$$

\section{References}

1. H. Bondi, M.G.J. van der Burg, A.W.K. Metzner, Proc. Roy. Soc. A 269, 21 (1962)

2. L. Herrera, A. Di Prisco, J. Ospino, J. Carot, Phys. Rev. D 91, $024010(2015)$

3. L. Herrera, A. Di Prisco, J. Ibáñez, J. Ospino, Phys. Rev. D 89, 084034 (2014)

4. G.F.R. Ellis, Relativistic Cosmology in Proceedings of the International School of Physics "Enrico Fermi", Course 47: General Relativity and Cosmology, ed. by R.K. Sachs (Academic Press, New York, 1971)

5. G.F.R. Ellis, H. van Ellst, (1998). arXiv:gr-qc/9812046 [v4]

6. G.F.R. Ellis, Gen. Rel. Gravit. 41, 581 (2009)

7. G.F.R. Ellis, R. Maartens, M.A.H. MacCallum, Relativistic Cosmology (Cambridge U. P., Cambridge, 2012)

8. L. Herrera, J. Ospino, A. Di Prisco, E. Fuenmayor, O. Troconis, Phys. Rev. D 79, 064025 (2009)

9. L. Bel, C. R. Acad. Sci. 247, 1094 (1958)

10. L. Bel, Cah. de Phys. 16, 59 (1962)

11. L. Bel, Gen. Rel. Grav. 32, 2047 (2000)

12. A.G.-P. Gómez Lobo, Class. Quantum Grav. 25, 015006 (2008)

13. L. Herrera, W. Barreto, J. Carot, A. Di Prisco, Class. Quantum. Grav. 24, 2645 (2007)

14. R. Sachs, Proc. Roy. Soc. A 270, 103 (1962)

15. L. Herrera, Gen. Rel. Grav. 46, 1654 (2014)

16. L. Herrera, A. Di Prisco, J. Ospino, J. Carot, Phys. Rev. D 94, 064072 (2016)

17. L. Herrera, N.O. Santos, J. Carot, J. Math. Phys. 47, 052502 (2006)

18. W.B. Bonnor, in Proceedings of the Meeting on General Relativity, ed. by G. Barbera. vol II (Florence, 1965)

19. W.B. Bonnor, in Ondes et Radiations Gravitationelles (Editions du Centre Nationale de la Recherche Scientifique, Paris, 1974) 